ARTICLE

\title{
Field test of wide-range environmental neutron dosemeter using PADC
}

\author{
Wakako SHINOZAKI ${ }^{1}$, Daisuke MAKI ${ }^{1}$, Hiroyuki OHGUCHI ${ }^{1}$, Masashi TAKADA ${ }^{2}$, Takashi NAKAMURA ${ }^{3}$ \\ and Takayoshi YAMAMOTO ${ }^{1}$
}

\author{
${ }^{1}$ Oarai Research Center, Chiyoda Technol Corporation, \\ 3681, Narita-cho, Oarai-machi, Higashi-ibaraki-gun, Ibaraki-ken, 311-1313, Japan \\ ${ }^{2}$ National Institute of Radiological Science, \\ 4-9-1, Anagawa, Inage-ku, Chiba, 263-8555, Japan \\ ${ }^{3}$ Cyclotron and Radioisotope Center, Tohoku University, \\ 6-3, Aoba, Aramaki, Aoba-ku, Sendai, Miyagi, 980-8578, Japan
}

\begin{abstract}
Environmental neutron dosemeter using PADC (Polly Allyl Di-glycol Carbonate) track detectors was tested in an actual neutron field. The dosemeter was set at a point in the radiation controlled area around the cyclotron facility of National Institute of Radiological Sciences (NIRS), together with the commercially available rem counter for comparison. For further validation of the dosemeter, the neutron spectrum at the point was measured by using Bonner ball counters inserting gold foil. The dosemeter showed the dose comparable to that estimated from the spectrum. The incident neutron direction was also estimated by detecting the slow neutrons from track densities on the PADC detectors.
\end{abstract}

\section{KEYWORDS: dosemeter, PADC, ambient dose equivalent, Bonner ball, unfolding}

\section{Introduction}

In Japanese regulation law, monitoring of ambient dose equivalent is required at the boundaries of radiation controlled areas and nuclear facility sites. For neutron monitoring, real-time dose equivalent counters, so-called rem counters, are widely used. The rem counters have high reliability as environmental neutron monitoring devices because of the energy response well fitted to the curve of ICRP74 fluence-to-ambient-dose-equivalent conversion coefficients ${ }^{1)}$ and the excellent directional response. However, in fact, some rem counters underestimate the dose in the neutron energy above about $10 \mathrm{MeV}$ and on the contrary overestimate drastically in the medium energy region of 10 $\mathrm{eV}$ to $10 \mathrm{keV}$. Besides the rem counters also have some demerits such as the heavy weight (about $10 \mathrm{~kg}$ ) and the high cost.

Our group has developed a new type of wide-range environmental neutron dosemeter ${ }^{2)}$ using PADC (Poly Allyl Di-glycol Carbonate) track detectors embedded in polyethylene moderator. The PADC track detectors are widely used as passive-type neutron detectors having the following features; good sensitivity to fast neutrons, small fading, light weight and low cost. By using PADC track detectors, our environmental neutron dosemeter can give the integrated value of neutron ambient dose equivalent for a long-term use. The dosemeter can also be used in gamma-ray and neutron mixed fields due to the insensitivity to gamma rays.

In this study, characteristics of the developed neutron dosemeter were investigated in an actual neutron field around

*Corresponding Author, E-mail:shinozaki-w@c-technol.co.jp

(C) Atomic Energy Society of Japan the cyclotron facility of National Institute of Radiological Sciences (NIRS) in Japan. The neutron ambient dose equivalent was measured using the dosemeter and a rem counter. The neutron energy spectrum and the ambient dose equivalent in this neutron field were also evaluated by the measurement of the multi-moderator spectrometer, so-called Bonner ball, inserting gold activation foil.

\section{Materials and Methods}

\section{Wide-range environmental neutron dosemeter}

The new type of wide-range environmental neutron dosemeter consists of a polyethylene block of the octagon pillar, $6 \mathrm{~cm}(\mathrm{~L}) \times 6 \mathrm{~cm}(\mathrm{H}) \times 6 \mathrm{~cm}(\mathrm{D})$, as a moderator and radiator, and eight PADC detectors (TechnoTrak; Chiyoda Technol Co.), $19 \mathrm{~mm}(\mathrm{~L}) \times 8.5 \mathrm{~mm}(\mathrm{H}) \times 1.6 \mathrm{~mm}(\mathrm{D})$, as neutron detectors. It weights only about $200 \mathrm{~g}$. Four PADC detectors are put at $1 \mathrm{~mm}$ depth of the polyethylene block to be used as fast neutron sensors detecting recoil protons produced by nuclear reactions with the polyethylene block and/or the PADC itself. On the other hand, other four PADC detectors with boron nitride (BN) radiator are put at $10 \mathrm{~mm}$ depth of the block to be used as slow neutron sensors detecting alpha particles produced by ${ }^{10} \mathrm{~B}(\mathrm{n}, \alpha)$ reactions. The PADC detectors are embedded in the block at intervals of 90 degrees as shown in Fig.1. Due to the configuration of the detectors, it is confirmed in our former study ${ }^{2}$ that the dosemeter has uniform sensitivity for incident neutrons from $2 \pi$ directions. The latent tracks formed in the PADC detectors by charged particles produced via the (n.p) and $(n, \alpha)$ nuclear reactions with $\mathrm{H}, \mathrm{C}$ and B nuclei are enlarged to visible size by chemical etching and then counted for dose estimation. Neutron ambient dose equivalent, $\mathrm{H}^{*}(10)$, is then estimated as follows. 

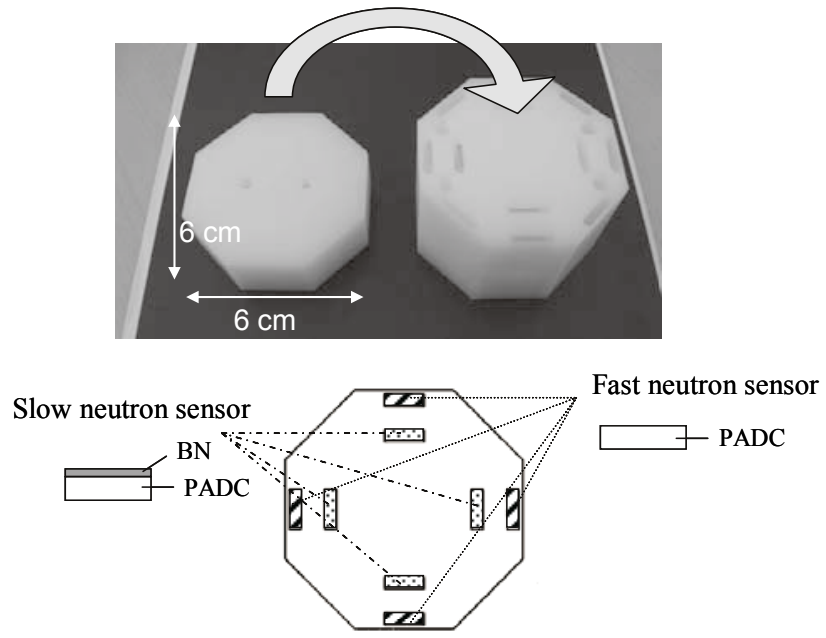

Fig. 1 External and the cross-sectional views of the wide-range environmental neutron dosemeter

$$
\mathrm{H}^{*}(10)(\mathrm{mSv})=\mathrm{a} \Sigma \mathrm{S}+\mathrm{b} \sum \mathrm{F},
$$

where, $\mathrm{S}$ and $\mathrm{F}$ are track densities $\left(\right.$ tracks $/ \mathrm{cm}^{2}$ ) for the slow and fast neutron sensors, respectively, and $\mathrm{a}$ and $\mathrm{b}$ are conversion factors from the track density to $\mathrm{H}^{*}(10)$. The factors were determined from irradiation tests in calibration fields and Monte Carlo calculation, which is described in detail in ref.2. Response curve of the dosemeter obtained from the calculation and experimental data using thermal neutron, some monoenergetic neutrons $(565 \mathrm{keV}, 5 \mathrm{MeV}$, $14.8 \mathrm{MeV}$ ) and ${ }^{241} \mathrm{Am}-\mathrm{Be}$ and ${ }^{252} \mathrm{Cf}$ neutron sources are shown in Fig.2 with the curve of fluence-to-ambient-dose-equivalent conversion coefficients in ICRP74. Responses of some rem counters ${ }^{3)}$ are also shown in the figure. They all are normalized to the response for ${ }^{252} \mathrm{Cf}$ neutrons (average energy; $2.3 \mathrm{MeV}$ ). It can be seen that the dosemeter has the closer response to the conversion coefficient than the rem counters especially in the energy region of $10 \mathrm{eV}$ to $100 \mathrm{keV}$.

\section{Field test}

An actual neutron field test was carried out around the cyclotron facility of NIRS when the cyclotron was in operation. Neutron ambient dose equivalents were measured by using the dosemeter and the rem counter (TPS-451BS) at a point in the radiation controlled area outside the cyclotron facility. Fig.3 shows a schematic view of the cyclotron facility and measurement point in the field test. Neutrons produced from the copper target bombarded by $30 \mathrm{MeV}$ protons were streamed through the maze and transmitted through the concrete shield to the measurement point in the radiation controlled area. The dosemeter was set for 3.5 hours. After the exposure, all PADC sensors in the dosemeter were chemically etched in $30 \mathrm{wt} \% \mathrm{KOH}$ at $90^{\circ} \mathrm{C}$. Etching times were 2.5 hours after the pre-soaking

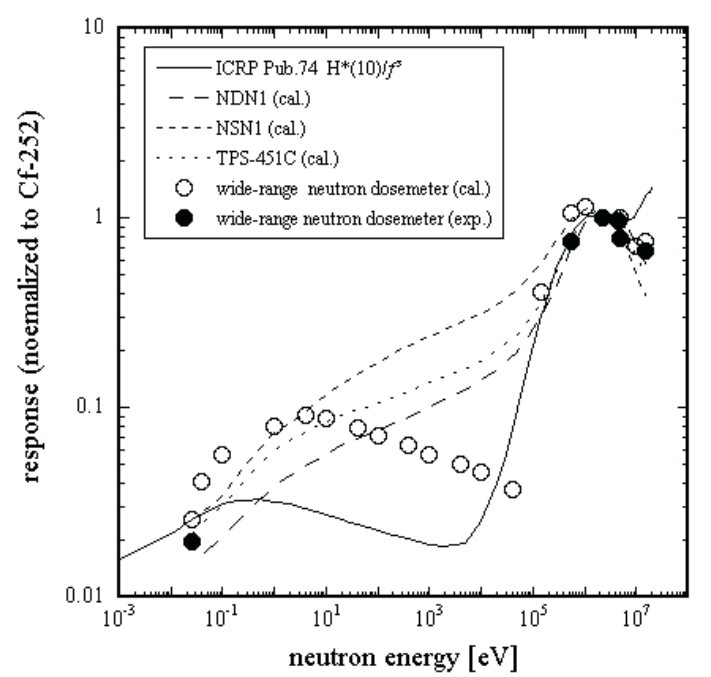

Fig. 2 Response curves of the dosemeter and some rem counters by comparing the ICRP74 fluence-to-ambient dose equivalent conversion coefficients

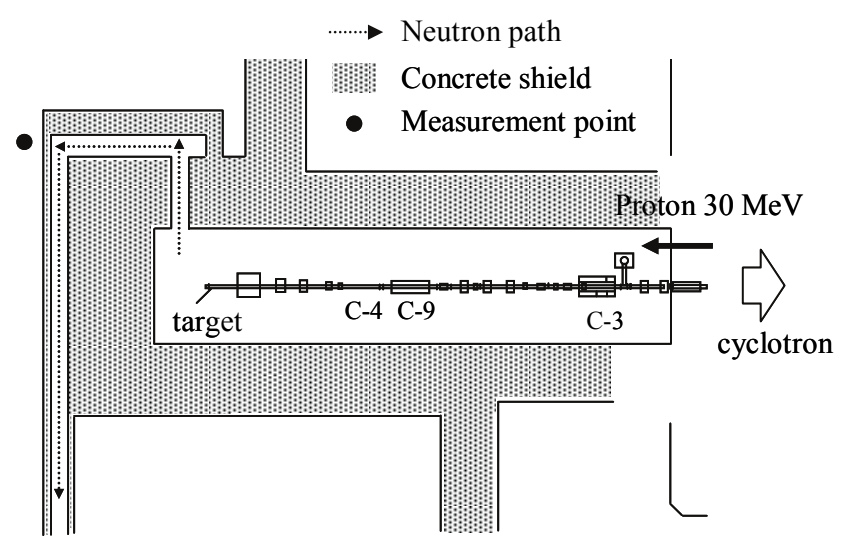

Fig. 3 Schematic view of the NIRS cyclotron facility and the measurement point in the radiation controlled area

procedure4) with DBP (dibutyl phthalate) for the fast neutron sensors, 15 minutes for the slow neutron sensors without pre-soaking procedure. After the etching procedure, recorded tracks on the PADC sensors were counted by image analyzing system with the automatic microscope (Hsp-1000CS5); SEIKO PRECISION INC.) and then neutron ambient dose equivalent was evaluated by Eq. (1).

In order to validate the dose estimated from the dosemeter more accurately, the spectrum at the measurement point was also measured by a spectrometry technique using the Bonner balls inserting gold activation foil. The bare gold foil and the Bonner balls with three different polyethylene moderators of $2.54 \mathrm{~cm}, 3.81 \mathrm{~cm}$, and $6.35 \mathrm{~cm}$ radii were exposed at the point. The $412 \mathrm{keV}$ gamma rays emitted from gold foils by ${ }^{197} \mathrm{Au}(\mathrm{n}, \gamma){ }^{198} \mathrm{Au}$ reaction were measured with a Ge detector. 


\section{Estimation of neutron energy spectrum}

Response functions for the three Bonner balls and bare gold foil were calculated by the MCNP4C2 code $^{6), 7)}$ for 47 energy bins used in ICRP74 fluence-to-ambient-doseequivalent conversion coefficients. Fig.4 shows the calculated response functions. A peak around $5 \mathrm{eV}$ in the response function of bare gold foil reflects the resonance of ${ }^{197} \mathrm{Au}(\mathrm{n}, \gamma){ }^{198} \mathrm{Au}$ reaction. As the polyethylene moderator thickness increases, the response function becomes higher sensitivity in higher energy region. To obtain the energy spectrum at the measurement point, the reaction rates of ${ }^{197} \mathrm{Au}(\mathrm{n}, \gamma){ }^{198} \mathrm{Au}$ measured for the three polyethylene moderators were unfolded with the SAND-2 code ${ }^{8}$. In this unfolding, response function of bare gold foil was not used because of the resonance peak of $\mathrm{Au}(\mathrm{n}, \gamma)$ reaction around 5 $\mathrm{eV}$. Thermal neutron flux was estimated separately from the response of bare gold foil under the assumption that activation rate of gold foil was induced only by thermal neutrons because we did not use the cadmium ratio method. The cross section of $\mathrm{Au}(\mathrm{n}, \gamma)$ reaction of 98.65 barn was used for thermal neutron. The 1/E spectrum, which is well known as neutron spectrum in the environmental field, was applied as an initial guess above thermal energy for the unfolding.

\section{Results and Discussions}

The neutron energy spectrum in the field obtained by the Bonner ball is shown in Fig.5. The spectrum is very soft and is close to $1 / \mathrm{E}$ spectrum below about $10 \mathrm{keV}$, but rapidly decreases above $100 \mathrm{keV}$. The fluence-weighted average energy of this neutron spectrum was $25 \mathrm{keV}$. The contribution of thermal neutrons was not included in the spectrum because the response function of the bare gold foil was not used for unfolding. The thermal neutron flux was estimated as $2.58 \times 10^{2} \mathrm{~cm}^{-2} \mathrm{~s}^{-1}$ from the response of the bare gold foil. This value is about three times of $1.00 \times 10^{2} \mathrm{~cm}^{-2} \mathrm{~s}^{-1}$ below $0.5 \mathrm{eV}$ obtained from the unfolding, although this value estimated without using cadmium ratio method gives a little overestimation.

From the estimated thermal neutron flux and the measured spectrum, the ambient dose equivalent rate at the measurement point in the field was evaluated as $17.2 \mu \mathrm{Sv} / \mathrm{h}$ by using fluence-to-ambient-dose-equivalent conversion coefficients in ICRP74. On the other hand, the ambient dose equivalent rate measured at the field test point was 14.3 $\mu \mathrm{Sv} / \mathrm{h}$ (ambient dose equivalent of $50 \mu \mathrm{Sv}$ integrated for 3.5 hours) by our wide-range neutron dosemeter and $18.2 \mu \mathrm{Sv} / \mathrm{h}$ by the rem counter. These results are shown in Table 1. The slight overestimation of the rem counter to the Bonner counter can be explained from the fact that the rem counter have higher response compared with the dose conversion coefficients in the medium energy region between $100 \mathrm{eV}$ to $100 \mathrm{keV}$, as seen in Fig.2. Our dosemeter showed a lower dose than that of the rem counter and Bonner ball. As one of the reasons for the underestimation, it is thought that the fast neutron sensors of the dosemeter were not used for dose estimation in this field test. Because of the very low neutron fluence above $100 \mathrm{keV}$, as seen in Fig.5, only about $2 \%$ of

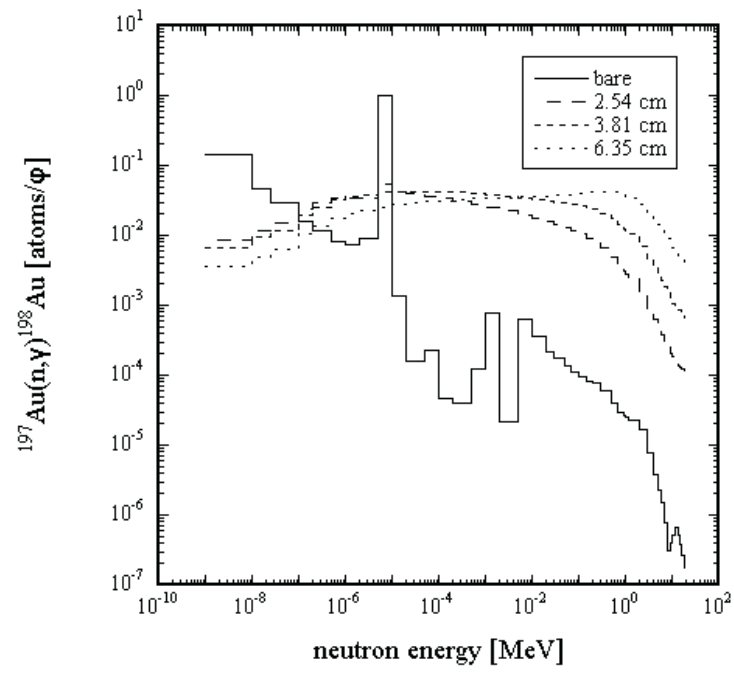

Fig. 4 Response functions of the Bonner counters obtained by the MCNP4C2 Monte Calro calculation

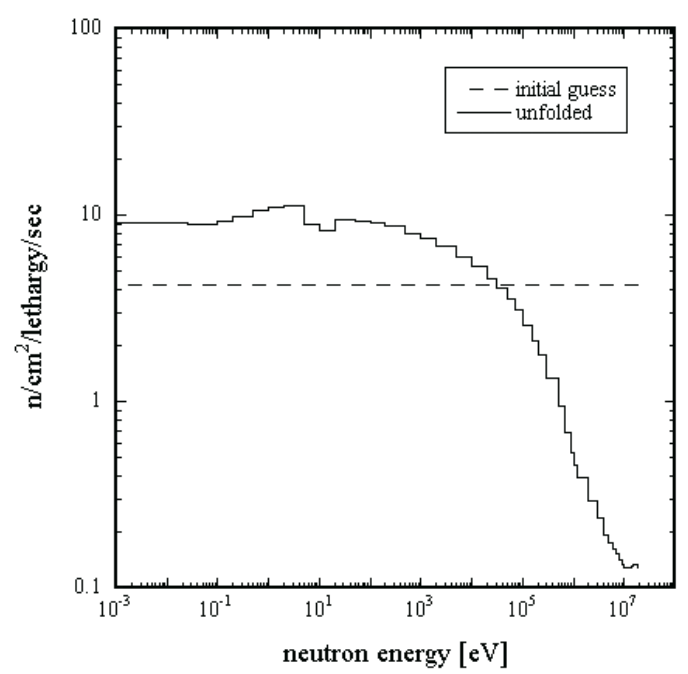

Fig. 5 Neutron spectrum at the field obtained by the SAND-2 unfolding

Table 1 The ambient dose equivalent rates measured by the dosemeter, the rem counter, and the Bonner counters.

\begin{tabular}{cc}
\hline detectors & $\begin{array}{c}\text { the ambient dose } \\
\text { equivalent rate }\end{array}$ \\
\hline $\begin{array}{c}\text { wide-range environmental } \\
\text { neutron dosemeter } \\
\text { rem counter }\end{array}$ & $14.3 \mu \mathrm{Sv} / \mathrm{h}$ \\
Bonner ball counter & $18.2 \mu \mathrm{Sv} / \mathrm{h}$ \\
& $17.2 \mu \mathrm{Sv} / \mathrm{h}$ \\
\hline
\end{tabular}

total neutron fluence during the 3.5 hours irradiation, tracks were hardly generated on the fast sensors and were less than $100 \mathrm{tracks} / \mathrm{cm}^{2}$. This value is a background level, that is the 
detection limit, on the characteristic of PADC using in the dosemeter ${ }^{9)}$. However in the actual use for monitoring the dose integrated over several months, the dose from the fast neutron sensors also will be able to be used even in the field where the fast neutron fluence is very low. The dosemeter will give higher dose if the contribution of fast neutrons is included for the dose evaluation in this test.

The relative response of the track densities of the four slow neutron sensors of the wide-range neutron dosemeter obtained by the experiment is shown in Fig.6. It was found that the sensor $\mathrm{S} 1$ faced to the concrete shield had the highest sensitivity and the sensor S3 at the opposite site of the $\mathrm{S} 1$ in the polyethylene block of the dosemeter had the lowest sensitivity. The sensor S2 and S4 showed almost the same sensitivities. It can be estimated from this result that a lot of thermal neutrons entered into the dosemeter in parallel directly from the shield. If thermal neutrons enter into the dosemeter from a certain direction, the sensitivities of the four slow neutron sensors should be different because the thickness of polyethylene that the neutron passes is different and if fast neutrons enter, it should be almost equal because the neutrons are uniformly distributed in the dosemeter by elastic scattering in polyethylene. The wide-range neutron dosemeter therefore may be able to estimate the incident direction of neutron for thermal neutrons. This information will be useful for monitoring the neutron field at the fixed point, such as the boundaries of radiation controlled area and facility site.

\section{Conclusion}

The new type of wide-range environmental neutron dosemeter was tested and compared with the rem counter in the neutron field around the cyclotron facility of NIRS. The ambient dose equivalent in the field was also evaluated using the Bonner ball counters inserting gold activation foil. As a result, it was found that the new dosemeter can give the ambient dose equivalent comparable to the calculated dose equivalent from the spectrum and the rem counter. In addition, it was also found that the information on incident neutron direction can be obtained from the distribution of track densities on four PADCs used as the slow neutron sensors. These results indicate that this dosemeter is useful as a detector for neutron monitoring at the boundaries of radiation controlled areas and facility sites.

\section{Acknowledgment}

The authors would like to their sincere thanks to the members of cyclotron facility in NIRS for their help of the experiment.

\section{References}

1) ICRP, Conversion Coefficients for Use in Radiological

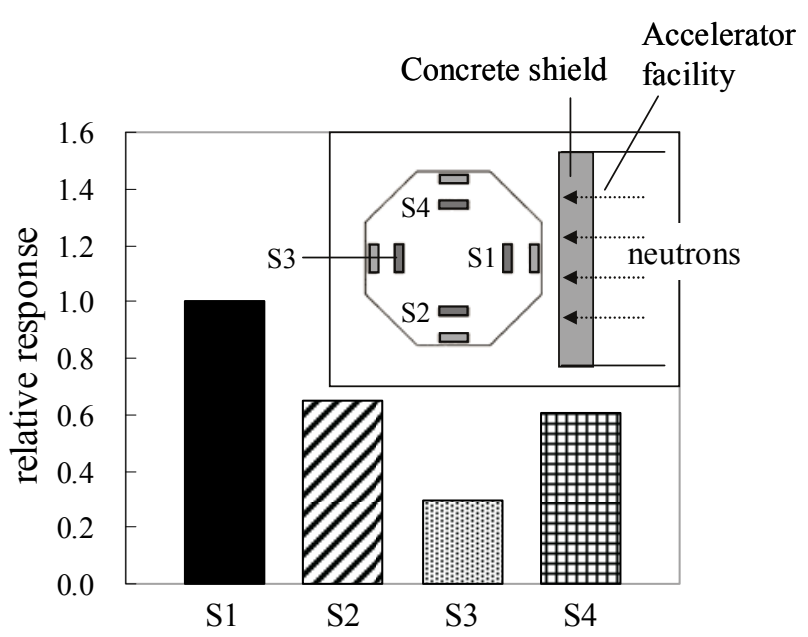

Fig. 6 Relative response of the track densities of the four slow neutron sensors of wide-range neutron dosemeter

Protection against External Radiation, ICRP Publication74, Annals of the ICRP, 26, No.3/4 (1996).

2) D. Maki, W. Shinozaki, H. Ohguchi, T. Nakamura, T. Yamamoto, Development of neutron dosemeter using CR-39 for environmental monitoring, Jpn. J. Health. Phys. To be submitted. [in Japanese]

3) J. Saegusa , M. Yoshizawa, Y. Tanimura, M. Yoshida, T. Yamano $b$ and H. Nakaoka, Evaluation of energy responses for neutron dose-equivalent meters made in Japan, Nucl. Instr. Meth., A, 516 1, 193-202(2004).

4) H.Ohguchi, K.Oda, T.Yamauchi, T.Nakamura, D.Maki; New pre-soaking technique for PADC and application to wide-range personal neutron dosimeter, Radiat. Meas, 43, 500-503(2008).

5) N. Yasuda, K. Namiki, Y. Honma, Y. Umeshima, Y. Marumo, H. Ishii, E.R.Benton, Development of a high speed imaging microscope and new software for nuclear track detector analysis., Radiat. Meas. 40,311-315(2005).

6) J.F.Briesmeister(Ed).,"MCNP-A General Monte Carlo N-Particle Transport Code Version 4C-Manual," LA-13709-M, Los Alamos National Laboratory, Los Alamos, New Mexico,USA, (2000).

7) ENDF-B/VI., Evaluated Nuclear Data File (ENDF-B/VI). National Neutron Cross Section Center, Brookhaven National Laboratory, (1990).

8) W.N.Mc. Elroy, S. Berg, T. Crockett, R.G. Hawkins, A Computer Automated Interative Method for Neutron Flux Spectra Determination by Foil Activation, Awfl-TR-67-41 Vol.1-4, Air Force Weapons Laboratory (1967).

9) H. Ohguchi, W. Shinozaki, K. Oda, T. Nakamura, T. Yamauchi, Characteristics of PADC detectors using new pre-soaking technique., Radiat. Meas. 43,437-441(2008). 